found to give rise to a slight local swelling and tenderness, lasting for about 24 hours.

Course of immunisation.-Patients received subcutaneous injections of pollen extract. At first very minute doses were given at intervals of three or four days (Fig. 1), and the resistance of the patients rose rapidly ; on increasing the dose, however, it was found that the resistance ceased to rise and even went back towards its original value. Longer intervals were then allowed to elapse between successive

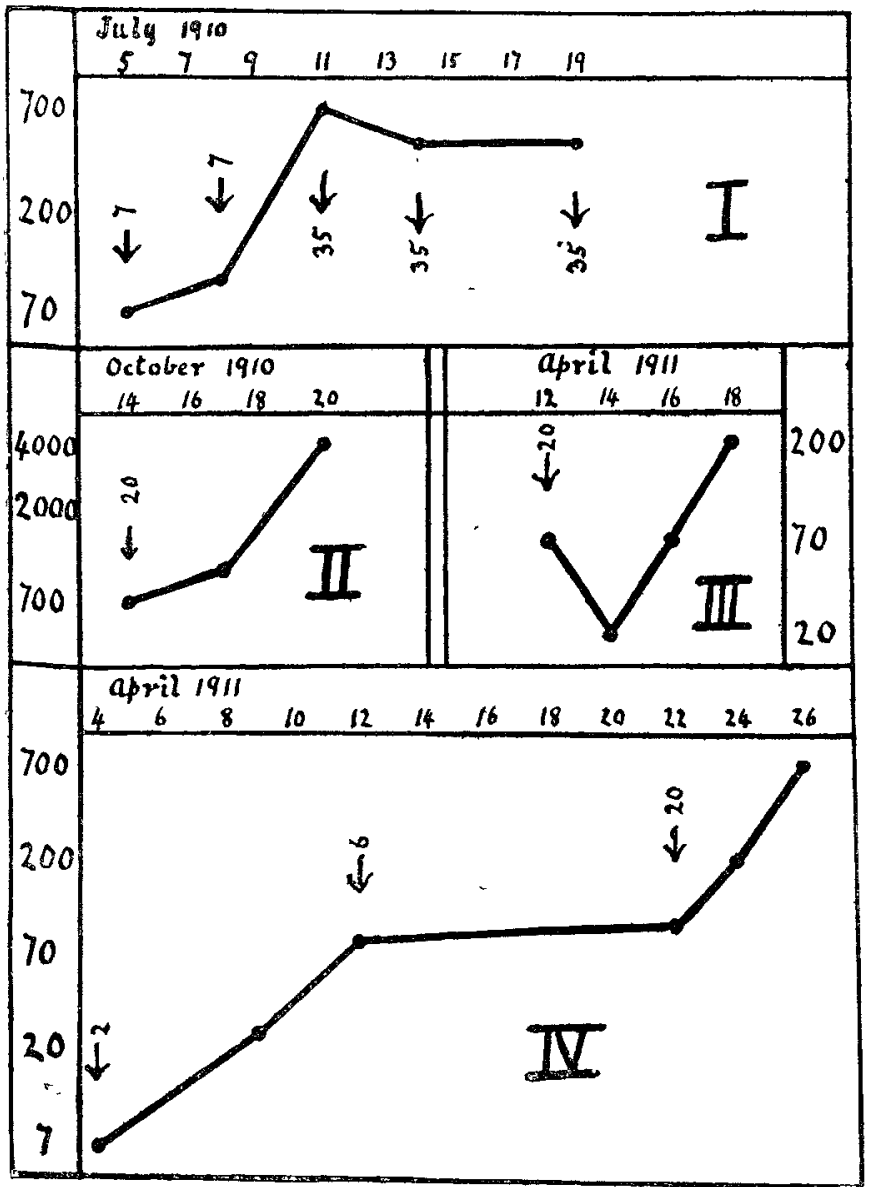

The numbers at the sides denote the resistance of the patient, given in terms of the strength of pollen extract, one drop of which was sufficient te excite a conjunctival reaction. The arrows indicate subcutaneous inoculations of pollen extract quantities given in the units described in the text. Figs. and 2 refer to one patient at different periods of treatment Fig. 3 shows the response obtained after about a month' treatment in another case; and Fig. 4 the early stages of treatment.

inoculations. The patient to whom Fig. 1 refers had a three months' respite and, after that interval, responded to a moderate dose in the way shown in Fig. 2 . It is not neces sary, however, to leave such a long interval as this between the doses : ten days or a fortnight are, as a rule, sufficient, and at the beginning of treatment, when small doses are being given, a week is enough (Fig. 4). After some time, when the resistance has been considerably raised, small doses cease to have any effect. On increasing the dose it is found that the first effect of the inoculation is to lower the resistance for a few days, giving a negutive phase, after which the resistance rises again and passes beyond its former maximum (Fig. 3). Ultimately a stage is reached at which the resistance, as measured by the ocular test, ceases to rise, or rises so slowly that the alteration is only obvious after prolonged observation. At this stage the patients will withstand gradually increasing subcutaneous inoculations without showing a negative phase. In the early stages of immunisation it is possible, by an overdose, to induce a severe attack of hay fever lasting nearly 24 hours; this has not been observed in the later stages.

The result of these experiments so far is to show that the sensibility of hay fever patients may be decreased, by properly directed dosage, at least a hundredfold, while excessive or too frequent inoculations only serve to increase the sensibility. It still remains to be seen whether the immunity thus attained is sufficient to carry the patients through a season without suffering from their annual attacks of hay fever. Patients are under observation who have underg one treatment for periods varying from a few weeks to eight months.

It is hoped that these cases will afford material for a further report after the present hay fever season. This work is now in the hands of $m p$ colleague, Dr. J. Freeman, who very kindly came to my assistance and carried on the observations during my enforced absence of some months.

Devonshire-place, w.

\section{SUPPURATIVE ARTHRITIS OF KNEE- JOINT, DUE TO INFLUENZA BACILLUS, OCCURRING IN A BABY AGED SIX MONTHS.}

By ELIZABETH T. FRASER, M.D. GLaSG., ASSIBTANT BACTERIOLOGIST TO THE GLASGOW ROXAT INFLRMARY.

IN the present indefinite state of our knowledge regarding the bacillus of Pfeiffer and allied species, each case where such organisms are found demands careful study. The following is the first recorded case of an uncomplicated mono-articular suppurative arthritis due to the bacillus influenzæ, and it presents several features worthy of note.

A healthy baby, aged 6 months, began on Nov. 13th, 1910 , to suffer from pain and swelling of the left knee-joint. She was first seen by a medical man on Nov. 19th, and, as she was feverish and the joint seemed much swollen, was at once removed to the Glasgow Royal Infirmary. The temperature on admission was $101 \cdot 6^{\circ} \mathrm{F}$. and the pulse-rate 128. On the 20 th the temperature was $102 \cdot 2^{\circ}$ and the pulse-rate 170. On the 21st the joint was incised and a considerable quantity of pus mixed with blood was evacuated; a drainage tube was left in. After operation the temperature fell to normal it afterwards went up to $100^{\circ}$. On the 27 th the temperature was $99.2^{\circ}$ and on the 29 th it was normal. The child made an uninterrupted recovery, at no time presenting any symptoms which were not attributable to the condition in the knee-joint.

The previous history of the case was carefully inquired into, and it was found that the child had never before suffered from any illness, that she had shown no trace of whooping-cough or influenza, and that neither in her family nor in the immediate neighbourhood was there any case of these maladies.

Baoteriology.--Stained smears from the pus showed chiefly polymorphonuclear leucocytes and a tiny bacillus resembling accurately the bacillus Pfeiffer. There was a considerable amount of phagocytosis. Cultures were made on various media. Those on ordinary agar and bouillon yielded no growth, but those on blood agar showed a growth resembling exactly that of bacillus Pfeiffer-minute transparent "dewdrop" colonies, which always remained discrete, and which had attained their maximum development in 24 hours. Microscopically the appearance conformed entirely to that of bacillas Pfeiffer-one found a tiny, slender, pleomorphic bacillus, tapered at one or both ends, show. ing many "diplococcal" forms and occasional so-called "filamentous" forms which measured five or six times the normal length. The organism was non-motile and Gram-negative; it stained feebly with the usual basic. dyes, such as methylene or toluidin blue. Besides growing on blood agar and blood bouillon the organism grew well on a "staphylococcus agar" made by melting a tube of ordinary agar and adding to it $\frac{1}{2}$ to 1 c.c. of a sterilised emulsion of staphylococci in saline solution. ${ }^{1}$ It is immaterial whether

1 This medium had been found by my colleague, Dr. A. Campbell, to be an excellent medium for bacillus Ducrey. In connexion with the growth of bacillus Pfeiffer, Grassberger had noted that on colonies of the former germ which might be in the neighbourhood colonies of the former germ which might be in the neighbourhood took on an exceptional development. Rosenthal had found that a dead culture of staphylococous had a similar "favourising" effect. If on sterilises an agar culture of staphy lococci in the autoclave and then pour it into a Petri plate and adds some blood to the medium one finds that it forms an exceptionally favourable culture ground for the bacillus Pfeiffer. Rosenthal considered that this "favourising" action was due to substances secreted by the staphylococei into the medium or to a modification of the medium under the influence of these microbes. Allen found that a bacillus influenze which refused to grow on ordinary blood agar could be got to grow when 2 c.c. of sterile bouillon culture of staphylococcus were added to it. The above-mentioned medium which we used required no addition of blood and did not require the presence of the medium on which the staphrlococcus had grown. The "favourising" action is here obviously due to the staphylococci them selves or to such products as are washed off from the surface of an agar slope by the salt solution. 
the emulsion be sterilised at $60^{\circ}$ or $100^{\circ} \mathrm{C}$. On this staphylococcus agar the bacillus grew more luxuriantly than on the blood media. The colonies were larger and less transparent. The organism also grew well on a streptococcus agar similarly prepared. The growth here was not so vigorous as on staphylococcus agar, but more so than on blood agar. The organism died out very quickly in cultures; it could not be subcultured on blood agar when older than two or three days. In blood bouillon it retained its vitality somewhat longer. In the original pus from the knee-joint (which contained a considerable proportion of blood) the germ remained alive in pure culture for 31 days.

Pathogencity.-Intravenous and intraperitoneal inocula. tions into the rabbit and intraperitoneal inoculation into the guinea-pig gave negative results. Inoculations into white mice of a culture from blood agar or blood bouillon similarly failed, but inoculation of an emulsion of the growth on a staphylococcus agar slope produced a septicæmia fatal in 24 hours. Pare cultures of the bacillus were obtained from the heart's blood, the liver, and the spleen. This power of producing septicæmia in a mouse and the fact that in the patient the bacillus must have travelled by the blood to reach the affected joint, are the only points in which this organism does not conform to the typioal bacillus of Pfeiffer.

One had now to ask oneself with Cohen: "Est-ce du bacille de Pfeiffer qu'il s'agit dans ce cas? Ou bien seulement d'un bacille identique d'aspect? Y aurait il autant de variétés de coccobacilles hémophiles qu'il y a de variétés de streptococques ou de bacilles acide-résistantes?" Cohen adds pathetically: "La solution de ce problème nous semble bien ardue."

Investigation of the literature shows no case similar to the above where a single joint was attacked by a bacillus resembling the bacillus Pfeiffer. Dudgeon and Adams report a case of a baby, 10 months old, with necrosis of the radius and suppuration of the elbow-joint followed by meningitis and abscess of the hip-joint. Post mortem the bacillus Pfeiffer was found in pure culture in the affected bones and joints and over the brain surface. Cohen has also found an influenza-like bacillus in the purulent fluid of joints in a case which died from cerebro-spinal meningitis. In both these cases there was a true septicæmia. Pfeiffer, Weichselbaum, Cantani, jun., Joachmann and others maintain that the bacillus is never found in the blood in the course of influenza. Canon, Meunier, and Ghedini, on the contrary, state that they have found it in the blood in these circumstances. Thursfield quotes two cases where he isolated typical influenza bacilli from the blood; both of these patients recovered.

An important contribution towards the elucidation of this question of the unity of the bacillus Pfeiffer has been made by Cohen in his report of three cases of septicæmic cerebrospinal meningitis. In these cases the organism was morphologically and culturally almost identical with the bacillus Pfeiffer, but when injected intravenously into a rabbit it killed the animal by a true septicæmia-not by toxæmia, as is the case with bacillus Pfeiffer. Cohen believes himself authorised to consider this microbe as representing an independent species.

In the present case we do not feel at liberty to claim the germ as belonging to a separate species. Although a septicæmia was produced in the mouse it is to be noted that this was possible only with the germ when grown on staphylococcus agar and not with the cultures on blood media, which had been those used by Pfeiffer.

Now Bordet has shown in his researches on the influenzalike bacillus found in whooping-cough that the characters of the "staphylococcus germ" (organism cultivated on sterilised agar which had previously been a culture medium for staphylococcus) differed in many respects from those of the "blood germ" (organism cultivated on blood media). "With regard to the agglutination reaction, the bacterium behaves as if it belonged to two different species depending upon the nature of the medium in which it is grown." The staphylococcus germ loses the power of being agglutinated by a serum which has a marked agglutinating action on the blood germ. It is quite rossible that in our inoculation experiments with white mice we were dealing with a "race résistante" (made resistant in vitro by the nature of the staphylococcus medium), and it would be quite unjustifiable to argue from this that the original germ was not bacillus
Pfeiffer. Until we know more of the possibilities of modifying the bacillas Pfeiffer by the action of various culture media, we shall not proceed far in our attempts to classify the influenza-like bacilli found in various pathological conditions.

I am indebted to Dr. J. A. Adams, under whose charge this case was admitted, and who very kindly furnished the clinical details. I am also much obliged to Mr. David McCrorie for performing the animal experiments necessary. Glasgow.

\section{A CASE OF ACUTE PHTHISIS FOLLOWING TYPHOID FEVER.}

BY W. SCARISBRICK, M.B., B.S., B.Sc. LOND., D.P.H., M.R.C.S. ENG., L.R.C.P. LOND.,

ASSISTANT MEDICAI OFFICER OF HEALTH OF THE CITX OF CHESTER.

ON Feb. 10th, 1911, a female patient, aged 13 years, was admitted to the Chester Isolation Hospital as a case of scarlet fever having the following history. Sore-throat and headache on Jan. 29th, but no vomiting, diarrhœa, or other symptom noticed until Feb. 9th, when the patient was thought to have scarlet fever desquamation. She was seen by me on the day of her admission to the isolation hospital, and the following notes were made: "Rash nil, tongue shows no special characteristic, throat normal. Suspicious denudation on the left heel observed, very like scarlet fever peeling, but no further signs of desquamation noted either at the time or subsequently. Heart and lungs normal; pulse and respiration 120 and 32 respectively, both showing an increase above the normal rate, ascribed, in view of the absence of signs in the chest, to excitement due to removal to hospital. Temperature $101.2^{\circ} \mathrm{F}$. Rose spots absent. No further signs noted."

On Feb. 14th the brother of the above patient was also admitted to the hospital as a case of scarlet fever, with signs and symptoms still more indefinite than those of his sister. This second case is still under observation, and I hope at a future date to record some interesting facts concerning the course of his illness.

Returning to the case of the girl, the subsequent history is interesting. On Feb. 14th it was noticed that the spleen was markedly enlarged, no rose spots were present (a sign which, in $\mathrm{my}$ experience, is rare in soung typhoid patients), and there were no signs of peeling. Some of the patient's blood was taken in order to test its agglutinative powers, but it was found to be negative to cultures of typhoid, paratyphoid A, and paratyphoid B. Nothing else worth recording was noted for the first few days of the patient's sojourn in hospital. On the 17th (a week after admission) the temperature dropped to normal for the first time since coming under our notice, and thereafter for the next week or so assumed an intermittent type, with the acme of the crest a little lower on each succeeding day, except one. In fact, the chart showed all the appearances of a case of enteric fever in the fourth week of illness, the temperature falling by lysis. During this period, and for the following fortnight, it was noted that the pulse and respirations varied very little from normal, the former only reaching 100 on one or two occasions and the latter rarely exceeding 20-24. The patient herself appeared, and in fact was, much improved, and complained of feeling hungry. A further sample of blood taken on Feb. 19th, and tested for Widal's reaction again gave a negative result. On the 25 th (a fortnight after admission) the temperature was normal and remained below $98^{\circ}$ for 24 hours. At this period a third sample of blood was taken, and on examination was now found to be positive to bacillus typhosus in dilutions 1 in 25 and 1 in 60 . In a dilution of 1 in 100 partial agglutination was also obtained. The diagnosis of typhoid fever, which might be said to have been doubtful previously, seemed now settled. On the next evening the temperature rose again and for the next week assumed a hectic type of increasing severity. The patient obviously had some kind of a relapse. She was restless and did not want her milk. The tongue was furred but moist. The respirations remained the same, 20 per minute, but the pulse gradually became more frequent, averaging 96 per minute on March 2nd. On this date the heart and lungs appeared to be normal, the spleen was palpable, and the 